\title{
Control of Stick-slip Oscillations in Oil Well Drill Strings Using the Back-stepping Technique
}

\author{
Mohamed Zribi \\ Department of Electrical Engineering, Kuwait University, P. O. Box 5969, Safat-13060, KUWAIT
}

Mansour Karkoub

Department of Mechanical Engineering, Texas A\&M University, Doha, Qatar

C. C. Huang

Department of Mechanical Engineering, Tatung University, Taipei, Taiwan

\section{(Received 6 September 2010, accepted 9 December 2010)}

This paper deals with the problem of suppression of stick-slip oscillations in oil well drill-strings. The backstepping technique is used to design two control schemes for the system. The first controller is a typical backstepping controller; the second controller is a sliding back-stepping controller. Moreover, a reduced order observer is proposed to estimate the unmeasurable states of the system. Simulation results of the two proposed control schemes with and without the observer, indicate that both control schemes work well.

\section{INTRODUCTION}

The drilling process is affected by the dynamically induced vibrations due to design imperfections as well as material elasticity. Because of these vibrations, premature wear and damage of drilling equipment may occur. Vibrations can decrease the rate of penetration (ROP), and thereby increase the cost of the well. ${ }^{1-3}$ Moreover, vibrations can interfere with measurementwhile-drilling (MWD) tools or even cause their damage. Another major problem caused by vibrations is induced well-bore instabilities which can worsen the condition of the well. ${ }^{4,5}$

The literature on drill strings can be classified into three groups: drill string modeling, drill string control, and drill string technology. In this paper, we will focus on drill string dynamics, and control. There exist a significant number of research publications on drill string dynamics and a representative few are considered here. Many different models for drillstrings are available in the literature that may include one or more of the following phenomena: bit-bounce, stick-slip, forward and backward whirl, axial, lateral, and torsional vibrations; see for example. ${ }^{6-9}$ Stick-slip is a major cause of torsional vibrations and many researchers tried to minimize its effect on the behavior of the drill string using active damping techniques. ${ }^{10-12}$ These techniques reduce the torque fluctuations and the torsional drill-string vibrations affecting in this manner the stick-slip conditions. The underlying concept is to reduce the amplitude of the downhole rotational vibrations using torque feedback. The feedback is used by the rotational drive which slows down the rotary rate when the torque increases and speeds it up when the torque decreases. ${ }^{8,13,14}$ Serrarens et al. ${ }^{15,16}$ used $H_{\infty}$ control to suppress stick-slip oscillations whereas Yigit and Christoforou ${ }^{17}$ used state feedback control for the same purpose. Hiddabi et al. ${ }^{18}$ developed a nonlinear controller to reduce the vibration of the drill string; the control law is based on input-output linearization and the authors showed, through simulations, the effectiveness of the proposed control law. Abdulgalil and Siguerdidjane ${ }^{19-21}$ developed a PID controller to reduce the effect of the torsional vibrations. The PID controller is used in conjunction with a state input controller because of the uncertainties of the system.

The sliding mode control technique has been widely used in many applications. One of the main reasons of the wide spread usage of this technique is its robustness with respect to changes in the parameters of the system and with respect to external disturbances; for example see. ${ }^{22-25}$

In this paper, we adopt a third order model of the drill-string system. We propose to use the back-stepping technique to control the system. A typical back-stepping controller as well as a sliding back-stepping controller are proposed to control the drill-string system. Moreover, since not all the states are available for feedback, an observer is designed for the system.

The paper is organized as follows. Section 2 presents a third order dynamic model of the drill string system. A backstepping controller for the drill string system is presented in section 3. A sliding back-stepping controller for the system is proposed in section 4 . In section 5 , an observer is proposed to estimate the unmeasurable states of the system. The simulation results are presented and discussed in section 6. Finally, the conclusion is given in section 7 . Sometimes, the arguments of a function will be omitted in the analysis when no confusion may arise.

\section{DYNAMIC MODEL OF THE SYSTEM}

Consider the drilling system represented in Fig. 1.

Let,

$\phi_{1}$ : the angular displacement of the bit, 